$\stackrel{N / N}{=}$

Global journals Inc.

है

\title{
Immune Status Creative Analysis in Patients after Coronary Artery Bypass Surgery (CABS) with the use of Cardiopulmonary Bypass (CPB) Machine
}

By Barsukov, A.A., Zemskov, V.M., Pronko, K.N., Kozlova, M.N., Shishkina, N.S., Demidova, V.S., Popov, V.A., Plotnikov, G.P., Korostelev, A.N., Kazennov, V.V., Zemskov, A.M., Sadiykov A.L. \& Revishvili A.Sh.

Abstract- An analysis of the immune status in 53 patients that underwent coronary artery bypass grafting under cardiopulmonary bypass was carried out in the preoperative period and on day 1 and 7 after the surgical intervention. Significant changes in innate and adaptive immunity were revealed. In the first case, they were expressed as an inflammatory process developed throughout the postoperative period; it was confirmed with an increase in leukocytes, total and stab granulocytes, monocytes, oxidative stress of phagocytes, CD64+ and CD40+ granulocytes, endogenous intoxication with the developed significant deficiency in CD4+ monocytes, and regulatory NK cells; while immediately after the surgery, it was confirmed by IgG and IgM levels. Some changes in adaptive immunity manifested through its activation, confirmed by an increased CD4+, CD11b+, HLA-DR+, and CD4+CD25+ lymphocyte content, together with a deficiency noted in total lymphocytes and CD8+ lymphocytes.

Keywords: CABS under CPB, immune system, immunovenin.

GJMR-I Classification: NLMC Code: WG 595
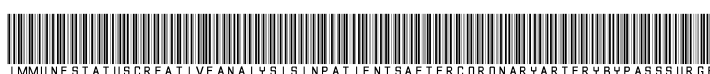

Strictly as per the compliance and regulations of:

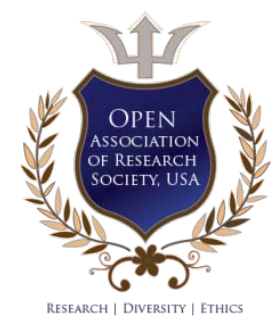

(C) 2020. Barsukov, A.A., Zemskov, V.M., Pronko, K.N., Kozlova, M.N., Shishkina, N.S., Demidova, V.S., Popov, V.A., Plotnikov, G.P., Korostelev, A.N., Kazennov, V.V., Zemskov, A.M., Sadiykov A.L. \& Revishvili A.Sh. This is a research/review paper, distributed under the terms of the Creative Commons Attribution-Noncommercial 3.0 Unported License http://creativecommons.org/licenses/by-nc/3.0/), permitting all non-commercial use, distribution, and reproduction in any medium, provided the original work is properly cited. 


\title{
Immune Status Creative Analysis in Patients after Coronary Artery Bypass Surgery (CABS) with the use of Cardiopulmonary Bypass (CPB) Machine
}

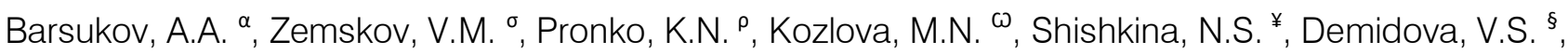 \\ Popov, V.A. ${ }^{x}$, Plotnikov, G.P. ${ }^{v}$, Korostelev, A.N. ${ }^{\ominus}$, Kazennov, V.V. ${ }^{\zeta}$, Zemskov, A.M. ${ }^{\ddagger}$, Sadiykov A.L. ${ }^{€}$ \\ \& Revishvili A.Sh. ${ }^{F}$
}

\begin{abstract}
An analysis of the immune status in 53 patients that underwent coronary artery bypass grafting under cardiopulmonary bypass was carried out in the preoperative period and on day 1 and 7 after the surgical intervention. Significant changes in innate and adaptive immunity were revealed. In the first case, they were expressed as an inflammatory process developed throughout the postoperative period; it was confirmed with an increase in leukocytes, total and stab granulocytes, monocytes, oxidative stress of phagocytes, CD64+ and CD40+ granulocytes, endogenous intoxication with the developed significant deficiency in CD4+ monocytes, and regulatory NK cells; while immediately after the surgery, it was confirmed by IgG and IgM levels. Some changes in adaptive immunity manifested through its
\end{abstract}

Author a: Senior Researcher of allergology and immunology, MD, of Clinical Immunology Group AV Vishnevski National Medical Research Center of Surgery, Moscow, Russia.

Author $\sigma$ : Professor of allergology and immunology, Ph.D, MD, Chief of Clinical Immunology Group AV Vishnevski National Medical Research Center of Surgery, Moscow, Russia. e-mail: arturrego@yandex.ru

Author p: Doctor of clinical psychology, Facecontrol, Systems, Moscow, Russia.

Author w: Senior Researcher of allergology and immunology, MD, of Clinical Immunology Group AV Vishnevski National Medical Research Center of Surgery, Moscow, Russia.

Author $¥$ : Junior Researcher of allergology and immunology, of Clinical Immunology Group AV Vishnevski National Medical Research Center of Surgery, Moscow, Russia.

Author \&: Doctor of biological sciences, Ph.D, MD, Chief of Clinical Laboratory Diagnostics AV Vishnevski National Medical Research Center of Surgery, Moscow, Russia.

Author $\chi$ : Professor of cardiac surgery, Ph.D, MD, Chief of Department of cardiac surgery AV Vishnevski National Medical Research Center of Surgery, Moscow, Russia.

Author v: Professor of anesthesiology and intensive care, Ph.D, MD, Chief of Department of Anesthesiology and Intensive Care AV Vishnevski National Medical Research Center of Surgery, Moscow, Russia.

Author $\theta$ : Professor of cardiac surgery, Ph.D, MD, Department of cardiac surgery AV Vishnevski National Medical Research Center of Surgery, Moscow, Russia.

Author ל: Doctor of anesthesiology and intensive care, Ph.D, MD, Department of Anesthesiology and Intensive Care AV Vishnevski National Medical Research Center of Surgery, Moscow, Russia.

Author f: Professor of allergology and immunology, Ph.D, MD, Chief of Department of Microbiology NN Burdenko Voronezh State Medical University, Russia.

Author €: Reseacher of Chromosystems Lab, Moscow, Russia.

Author F: Academician of RAS, Professor of cardiac surgery, Ph.D, MD, Director AV Vishnevski National Medical Research Center of Surgery, Moscow, Russia. activation, confirmed by an increased CD4+, CD11b+, HLA$\mathrm{DR}+$, and CD4+CD25+ lymphocyte content, together with a deficiency noted in total lymphocytes and CD8+ lymphocytes. These data are deemed to be fundamentally important since they were found before the patients were discharged and could persist in the future while being associated with an unknown outcome to be investigated in the long run. Immediately after a surgical intervention to a limited number of patients, the officinal IgG preparation (Immunovenin) was administered by six intravenous infusions to exert a corrective effect (mainly on the innate immunity) that was truly more significant than that in patients not having received the drug. By the time of discharge, patients have a reduced content of leukocytes, granulocytes, monocytes, CD40+ granulocytes, endogenous intoxication, together with the number of patients suffering from IgG deficiency reduced. It restored the initial level of sharply increased stab granulocyte content. The significance of the data obtained is being discussed.

Keywords: CABS under CPB, immune system, immunovenin.

\section{INTRODUCTION}

T he existing paradigm of the negative effect exerted on the immune system against the background of a heart and blood vessel pathology is beyond dispute [1]. Studies carried out in this matter have shown a significant effect exerted on the cardiovascular system status by immune cells. The main participants of the "negative process" were found to be innate immunity cells (i. e. neutrophils, monocytes) and adaptive immunity [T-lymphocytes, etc.] $[2,3]$.

Significantly, neutrophils and monocytes represent a heterogeneous population of cells, consisting of several subpopulations, differentiated by their functional properties $[4-7,9]$. Moreover, being the main cells of innate immunity, neutrophils function in two aspects relative to the cardiovascular system.

First, neutrophil in myocardial infarction promotes the transformation of M1 macrophage subpopulation (with pro-inflammatory properties) into M2 macrophage subpopulation (with anti-inflammatory and regenerative functions) [8], becoming an accomplice in the myocardial cell function recovery.

Secondly, neutrophils have significant cytotoxic potential, thus providing methods to damage the blood 
vessel endothelium [10] and cardiomyocytes [11]. A similar aggressive action attributed to neutrophils can be expected during heart surgery, especially in the case of CABS under CPB. Therefore monitoring of this process is required, as well as an in-depth selection of immunotropic drugs to level the pathological reaction.

At the same time, monocytes also consist of several subpopulations with different functional properties affecting the vessels and heart in different ways $[12,13]$. These are "classical" ones showing high pro-inflammatory, phagocytic, and antitumor activity, together with the production of oxygen radicals. "Intermediate" ones are those, featuring high phagocytic and pro-inflammatory activity, producing oxygen radicals, participating in the angiogenesis and proliferation of T cells; and "non-classical" antiinflammatory ones are those having virtually no phagocytosis and producing no oxygen radicals; they monitor the state of blood vessels, wound healing, and damaged tissues, tumorigenesis, and determine the stimulation and proliferation of $T$ cells [14]. Otherwise, they have the opposite effect.

Therefore, based on these considerations, the analysis of monocyte subpopulation in patients is of undeniable importance for studying the pathogenesisrelated to various diseases, including heart and blood vessel pathologies [13, 15-20]. The studies are not given in this paper. However, we paid great importance to them and are currently studying this problem intensively.

Even though the violation of adaptive immunity during cardiac surgery (incl. CABS, etc.) is also reflected in the literature [21-24], its nature is nevertheless multidirectional, which is especially true for open-heart surgery under cardiopulmonary bypass. Moreover, in this aspect, many issues regarding the state of the immune system, the nature of its disorders, and the correlation between the indicators attributed to innate and adaptive immunity are not well studied.

For the reasons mentioned, we focused on the study of congenital and adaptive immunity in cardiac operations under cardiopulmonary bypass, since in the patients having undergone surgery, the early postoperative period is accompanied by an aseptic systemic inflammatory response, which is 6 to $20 \%$ can initiate organ failure with a high mortality rate. The main goal of our research was to study the key cells of the mentioned immunity types with their expanded phenotypic and functional characteristics, some of which have not yet been studied. Also, attempts have been made to correct immune disorders using some immunotropic drugs.

\section{Materials and Methods}

Fifty-three patients, aged $48-77$ years, were enrolled in the study. The CPB time was 108 minutes
(71-168 minutes), the aortic clamping time was 51 minutes (33-84 minutes).

The blood for leukocytes was collected in tubes with $\mathrm{K}_{3} E D T A$, and for immunoglobulins, in the specific tubes, which were then centrifuged to obtain the serum.

Leukocytes phenotyping was performed on a FACSCalibur flow laser cytofluorimeter (BD, USA). The antibodies for the leukocytes membrane antigens analysis were obtained from BD Biosciences, Becton, Dickinson, San Joe, CA, USA, Beckman Coulter in the following range: CD3, CD4, CD8, CD11b, CD14, CD16, CD21, CD25, CD45, CD64, CD40, HLA-DR. CD56 and isotypic controls, FITC, and PE, were purchased from $\mathrm{BD}$, USA.

The cells were incubated with the antibodies in whole blood in the dark for 30 min at $40 \mathrm{C}$; then it was lysed with a BD FACS lysing solution and washed by centrifugation.

The analysis of the immunoglobulin isotypes (IgG, IgA, IgM) was performed using the monospecific antiserum manufactured by BioSystems (Spain), by the turbidimetric method on a semiautomated biochemical analyzer Screen Master Plus (Hospitex Diagnostics S. A., Sesto Florentino, Italy) at the wavelength of $340 \mathrm{~nm}$. Oxygen metabolism in phagocytic cells was analyzed (on a multiple Synergy 2 SLAD detector-analyzer, Winooski, Vermont, USA) with chemiluminescence, adding luminol or lucigenin used to detect generated reactive oxygen species (ROS) to enhance the chemiluminescence reaction. The reaction was conducted in wells in volume $150 \mu \mathrm{L}$ of luminol or lucigenin solution, $25 \mu \mathrm{L}$ of suspension of opsonized zymosan, and $5 \mu \mathrm{L}$ of whole blood of patients. Four repeated blood samples were used. Oxygenation in the presence of luminol supports the determination of the intracellular generation of a reactive oxygen intermediate [25]. In the case with the presence of lucigenin only, the extracellular generation of superoxide oxygen anion was detected [26].

Immunovenin was used in a group of patients $(n=18)$, at a dose of $50 \mathrm{ml}$ intravenously $(2.5 \mathrm{~g}$ of protein), six infusions in a row.

GraphPad Prism 7 software was used for the statistical analysis. The paired and unpaired t-tests were used in work. The data obtained are presented as mean value \pm standard error of the mean. Pearson's $\chi^{2}$ criterion was also used. The results were considered reliable at $\mathrm{p}<0.05$ or less.

This study is not a clinical trial. Therefore, all items do not require a response, since the studies were carried out within the framework of permitted surgical and immune methods. Sponsors did not participate in the design, execution, interpretation, or writing of the research or writing. All subjects gave their informed consent to inclusion before they participated in the study. All clinical, laboratory, and immune studies were 
carried out under the ethical standards of the Helsinki Declaration. The study protocol contained ethical aspects and information on how the principles of the Helsinki Declaration are ensured.

\section{ili. Results And their Discussion}

a) Quantitative assay of innate immunity cells in patients after $C A B S$ under $C P B$

An extended analysis of cell markers (37 cell antigens) in patients after CABS under CPB was performed. The selected parameters reflect the main innate and adaptive immunity characteristics and reliably reveal one's immune status. Differences in immune parameters were determined by comparing the immune values of markers 1 and 7 days after surgery with preoperative indicators. Note that almost all immune parameters in the preoperative period did not significantly differ from the physiological norms. Therefore, the problem of any concomitant diseases was not of great significance, since otherwise, it would have significantly affected the immune status indicators.

At the beginning of the study, it was established that in patients after CABS, the bloodstream innate immunity cell number was significantly increased. Figure 1 shows the dynamics of changes in leukocytes (A), neutrophils $(B, C)$, and monocytes $(D)$ in patients before surgery, on days 1 and 7 after it. As it turned out, the number of leukocytes increased sharply on the 1st day after the surgery $(p=0.0001)$ and remained high until the seventh day compared to the preoperative value $(p=0.0001)$.

The analysis of neutrophils - granulocytes and stabs in patients after CABS under CPB showed a significant increase in cells in the peripheral blood on days 1 and 7 (Fig. 1B, C), the absolute level of monocytes during the analysis periods also significantly exceeded its preoperative values $(p=0.0001)$.
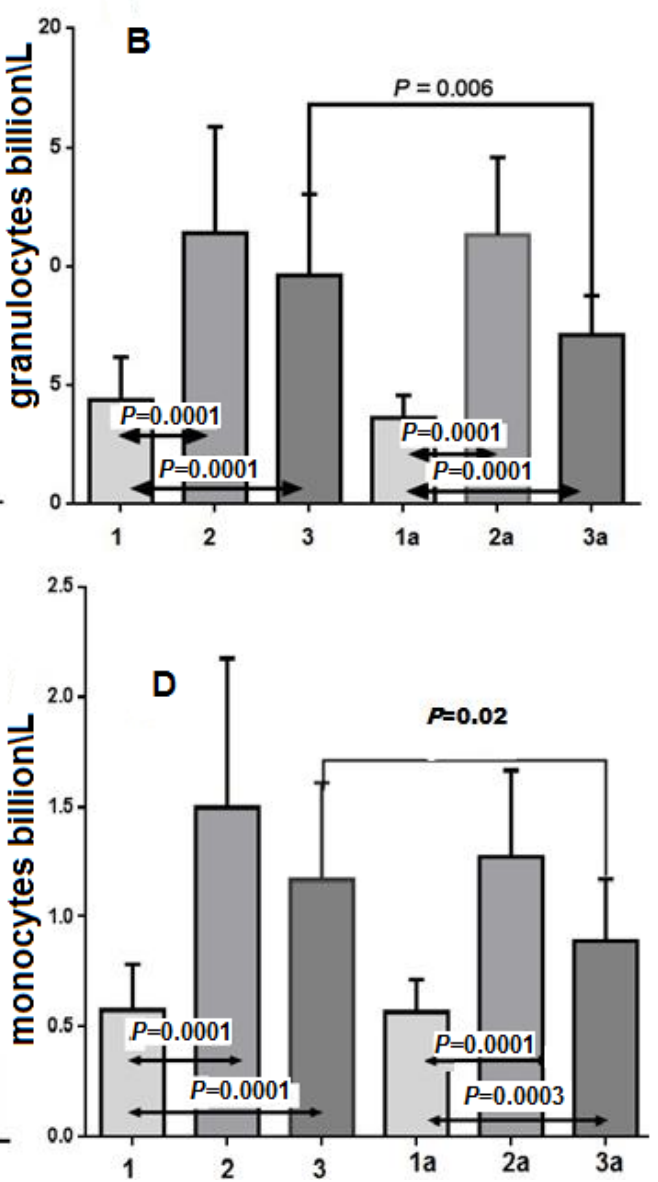

Figure 1: Increase in leukocytes (A) and innate immunity cells levels $(B, C, D)$ in patients after CABS under CPB. 1 - before surgery, $2-1$ day after the surgery, $3-7$ days after the surgery. Abbreviation

- a - patients who received Immunovenin

The absolute value of monocytes in the peripheral blood flow in patients after CABS under CPB (Fig. 1D) also significantly exceeded the value before surgery by the $1^{\text {st }}(p=0.0001)$ and $7^{\text {th }}$ days $(p=0.0001)$. b) Quantitative assay of cell-surface antigens in patients after $C A B S$ under $C P B$

The use of various markers (antibodies) is widely applied in the immune status monitoring in heart 
surgery [21]. First of all, the most important is the monitoring of neutrophils and the monocytic population of phagocytes, since, during heart surgery, their activity is often negative In our studies, we further showed significant changes in the functional properties of neutrophils and monocytes in patients after CABS. In Fig. 2, the results reflecting these changes are represented. As it turned out, on the 1st day after the surgery, the number of neutrophils with the membrane high-affinity $\mathrm{FC}_{\gamma}$ receptor (CD64) expression increased significantly. It is significant that the conditions after the surgery were sterile, which most likely reflects the stress activation of the cell (Fig. 2A). On day seven, this marker returned to the preoperative level $(p>0.05)$.

The functional activity of phagocytes also changed ("phagocytes" means the bulk of neutrophils with a small component of $\mathrm{Mn}$, as it is observed in the blood because in this way the 02-metabolism of blood cells is evaluated in total) - the intracellular oxygen metabolism was significantly enhanced during phagocytosis of opsonized zymosan 24 hours $(p=0.003)$ after the surgery (Fig. 2B). It also remained increased on day seven after the surgery $(p=0.005)$. It should be noted that before the surgery, in this category of patients, there was increased production of oxygen radicals (the upper norm is $16.4 \mathrm{EU} / 10000$ cells, and the value was $18.0 \mathrm{EU} / 10000$ cells in the patients).

For the first time, a small increase in neutrophils with CD40 receptor expression was found in patients after CABS after the surgery. However, they significantly increased on day 7 in the postoperative period $(p=0.009)$. This observation demonstrates the activity of neutrophils and their readiness to participate in the adaptive type immune reaction, and, probably, increased production of $\alpha$-TNF with cells $[5,6]$.
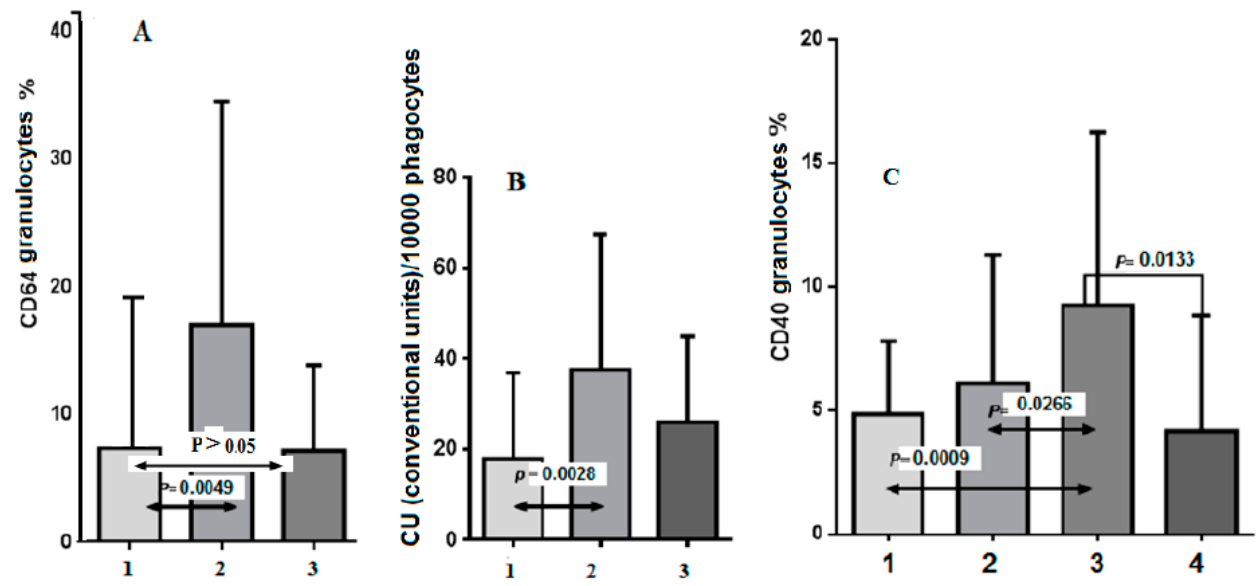

Figure 2: An increase in the number of neutrophils with CD64 (A) and CD40 (C) markers expression and the production of active oxygen radicals by phagocytes ( $\mathrm{B}$ - shown in equivalent units per $10^{4}$ cells) in patients after CABS under CPB.

1 - before surgery, 2 - 1 day after the surgery, 3-7 days after the surgery, 4 - patients are receiving Immunovenin.

Unexpected results were found when analyzing the expression on neutrophils of the low affinity $\mathrm{FC}_{\mathrm{C}}$-RIII receptor (CD16+ killer K-cells of neutrophils) in patients after CABS under CPB, whose role is to form the «gG + antigen» complex with its subsequent absorption by phagocytes, as well as the destruction of damaged lgG-bearing cells since it is also associated with the cytotoxic properties of a cell. We established the CD16 marker expression defect on neutrophils (Fig. 3). When studying the CD16 expression on neutrophils in patients on days 1 and 7 after the surgery, we established its significant change. So, in Figure 3 it can be seen that one day after the surgery, the histogram profile reflecting the affinity of FC-gamma RIII-receptors significantly changes on the phagocytes compared to the preoperative level and remains "inferior" on the $7^{\text {th }}$ day, which in some cases can contribute to the bacterial infection spreading. The significance of the discovered phenomenon for anti-infective organism protection has not yet been determined, but most likely, it is still negative. It should be noted that this phenomenon is not described in the literature. 
A

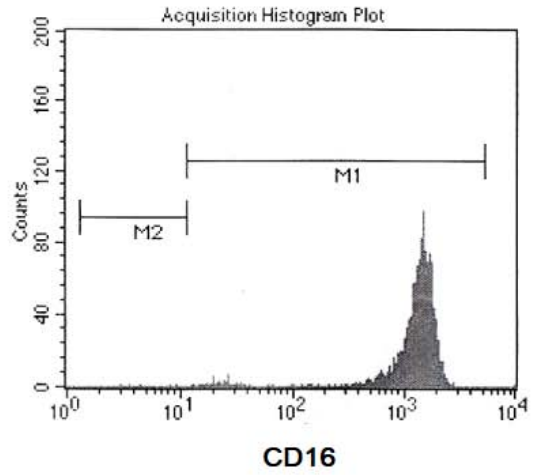

B

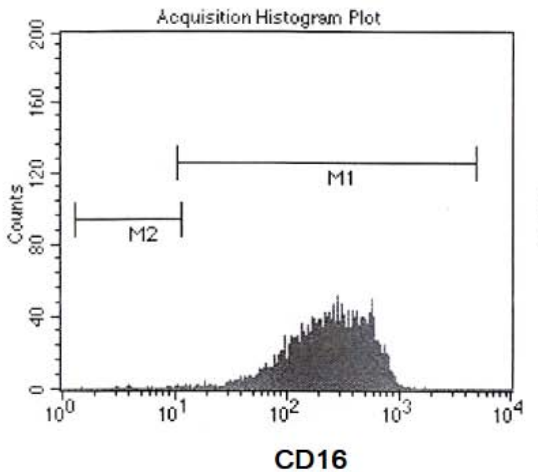

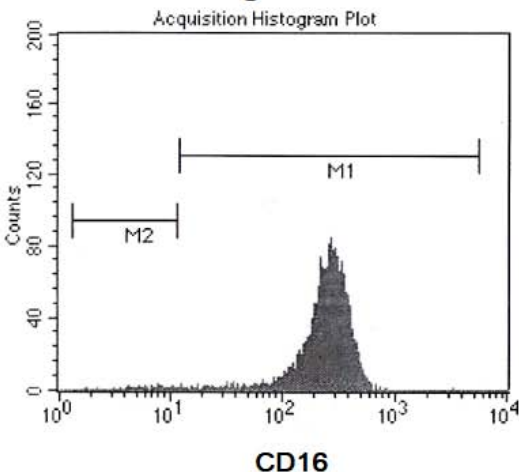

Figure 3: Neutrophilic CD16 marker expression change in patients after CABS under CPB.

A-before surgery; B- 1 day after the surgery; C - 7 days after the surgery.

It should be noted that in some cases, the expression of CD35 marker (CR1 complement immunoadhesin receptor) in patients after CABS under $\mathrm{CPB}$ also changed. However, this phenomenon was not observed in all patients, a y nine from 53 (19,68\%), compared to the expression of CD16 marker, which was constantly changing in all 53 the operated patients (Fig. 4).
A

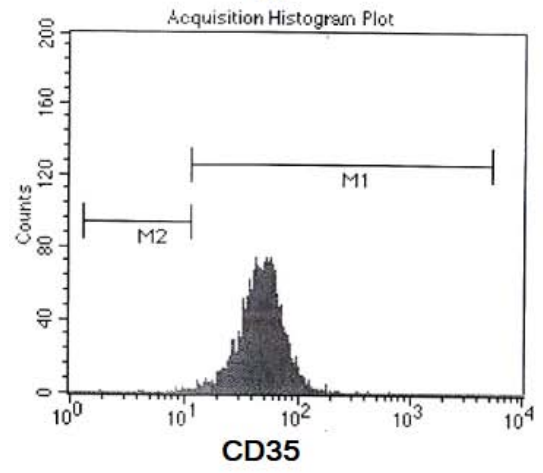

B

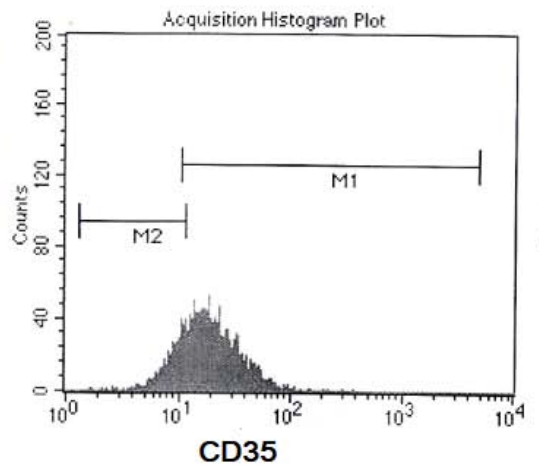

C

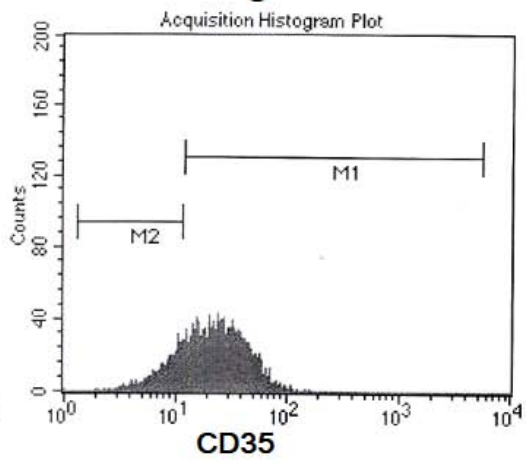

Figure 4: CD35 (CR1-complement receptor) expression change on neutrophils in patients after CABS under CPB.

A-before surgery; B- 1 day after the surgery; C- 7 days after the surgery.

Monocytes are the second most important and functional active innate immunity cells, although their number in circulation is much less than that of neutrophils (from 7 to 10\%). Their significance in cardiovascular pathology is confirmed by numerous studies. Monocytes, like neutrophils, participate both in "positive" reactions and in "negative" ones in case of a cardiopathy. In this work, it is clearly shown that in patients after CABS under CPB, there is a change in the number of monocytes with the expression of membrane antigens. Figure $5 \mathrm{~A}$ shows a significant increase in the number of monocytes with CD16 membrane marker (low-affinity $F c y$-RIII) expression one day after the surgery with normalization by day seven and a significant decrease in HLA-DR+ monocytes, returned to a normal value on day 7 (Fig. 5C).

On the other hand, CD56+ monocytes sharply increased immediately after the surgery, but also normalized on day 7 (Fig. 5D). Most likely, these dynamics are due to surgical intervention and a developed inflammatory response. In any case, a decrease in the number of HLA-DR + monocytes and an increase in CD56+ monocytes reflects particularly the inflammation development [29,34,35].

For the first time, we conducted a study of the CD4 monocytes content in patients after CABS under CPB. It showed a significant decrease in cells on the first day and seven days after the surgery (Fig. 5B). Unfortunately, little work has been devoted to the study of CD4 monocytes, and their role in immune regulation has not been studied. However, interesting studies on the role of CD4 monocytes are provided in [29]. Thus, it was shown that activation of the CD4 marker on cells through interaction with the major class II histocompatibility complex (MHC-II) triggers the production of cytokines and the differentiation of monocytes into functionally mature macrophages. Therefore, we can assume that the decrease in CD4 monocytes content that we observed in patients after CABS under CPB is a negative factor. 
Therefore, HLA-DR and CD4 membrane molecules can be apparently and fairly attributed to stress markers of monocytes. To a certain extent, this is confirmed by developed similar cellular changes in aseptic conditions of the postoperative period.
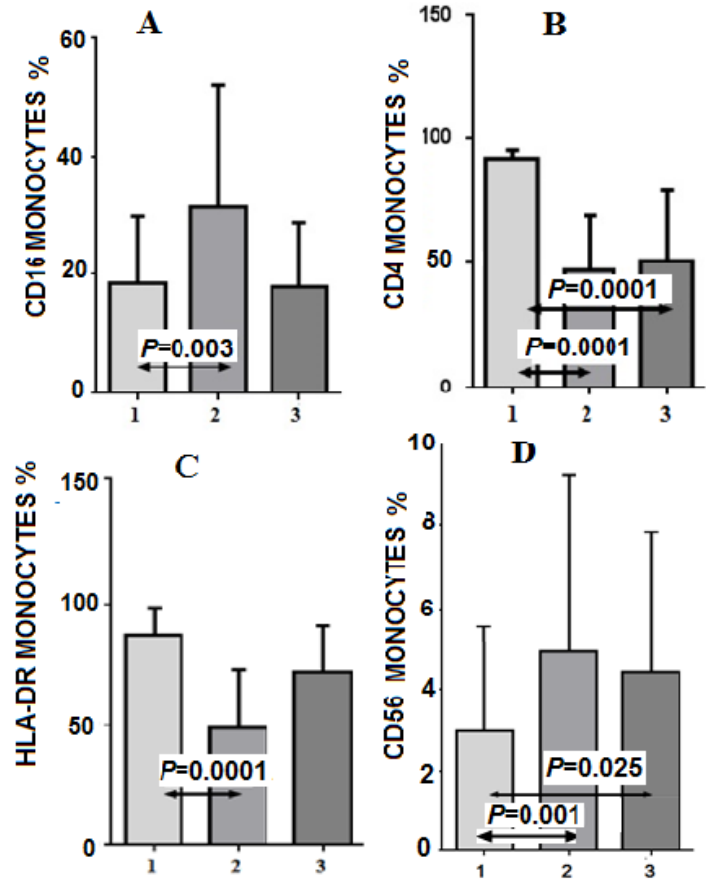

Figure 5: Change in monocytes content with the expression of CD16 marker (A), CD4 (B), HLA-DR+ antigens (C), and CD56 marker (D) in patients after CABS under CPB.

1 - before surgery, 2 - 1 day after the surgery, 3 -7 days after the surgery.

The revealed increase in the content of killer monocyte cells having expressed $\mathrm{Fcy}_{\mathrm{C}}$ receptors III (CD16+) [Fig. 5A] also possibly reflects an increase in the bactericidal properties of monocytes and their enhanced destruction of damaged cells immediately after the surgery with complete restoration of their function by day 7 of the postoperative period.

A study of natural killer effectors (CD56+CD16 + NK-effectors) belonging to the cells of innate immunity, demonstrated their increase in patients on day one after the surgery (Fig. 6). So, before the surgery, the relative content of CD56+CD16+ NKeffectors was $13.0 \pm 1.2 \%$, and after 24 hours after the surgery, the relative content was $17.0 \pm 1.3 \%$ $(p=0.038)$. This fact can be considered as positive, since natural killers of this type participate in many protective reactions of the body, including controlling cytodifferentiation, contributing to wound healing, and killing tumor cells. On the 7th day after the surgery, the relative number of these cells sharply decreased compared to the preoperative level $(p=0.0001)$, which already indicates the negative functional state of natural killer effectors, which might persist after discharging the patient, indicating negative changes in the cell killer system.

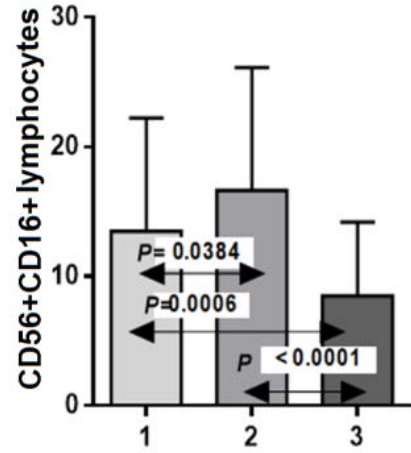

Figure 6: Change in the relative content of natural killer effectors CD56+CD16+ (\%).

1 - before surgery, 2 - 1 days after the surgery, $3-7$ days after the surgery.

c) Dynamic analysis of adaptive immunity cell changes in patients with $C A B G$ and IR

This section provides the results of studies of lymphoid cell membrane antigens related to adaptive immunity. Fig. 7 (A-C) indicates that, after a day in the surgery site, the content of total lymphocytes and their subpopulations (CD4-T-helpers/inducers, CD8cytotoxic/suppressor T-lymphocytes, and CD3-common T-cells). 

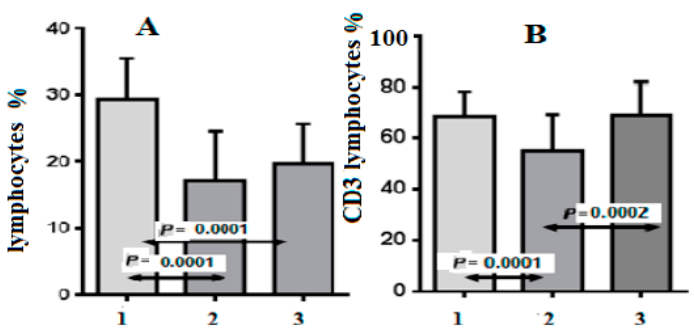

C
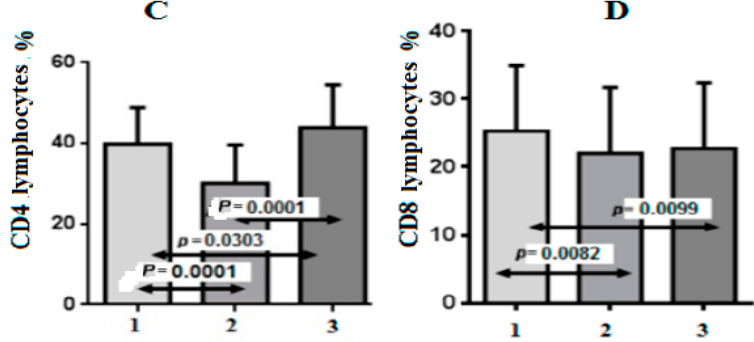

Figure 7: Changes in the relative content of total lymphocytes (A) and T-cells (B), T-helpers (C), and cytotoxic Tlymphocytes (D) in patients after CABS under CPB.

1 - before surgery, 2 - 1 days after the surgery, 3 -7 days after the surgery.

Underwent severe suppression, which most likely reflects their response to surgical trauma. By the time when patients were discharged (mostly, on day 7), the pattern was, however, different. A deep decrease in the content of total lymphocytes (7A) and cytotoxic/ suppressor T-lymphocytes (7D) was noted up to the day of discharge and, possibly, continued further; this is an undoubtedly negative factor regarding the alleged subsequent development of clinical immunodeficiency. At the same time, an increase $(p=0.03)$ of Thelper/regulatory lymphocytes by day seven may be a compensatory reaction of the immune system in response to CD8 T-lymphocytes suppression. In any case, this justifies a required examination of the patients that underwent surgery even after they are discharged with any possible complications stated.

Thus, the situation is traced in which specific lymphoid populations respond differently to stressful conditions caused by the surgery, that must be taken into account in cardiac surgery practice regarding CABS under CPB.

The purely opposite (compared to T-cells) change in the population of B-cells during heart surgery is noteworthy: on the first day, there was its significant increase $(p=0.0001$; Fig. $8 A$ ) with recovery occurred on day 7 .

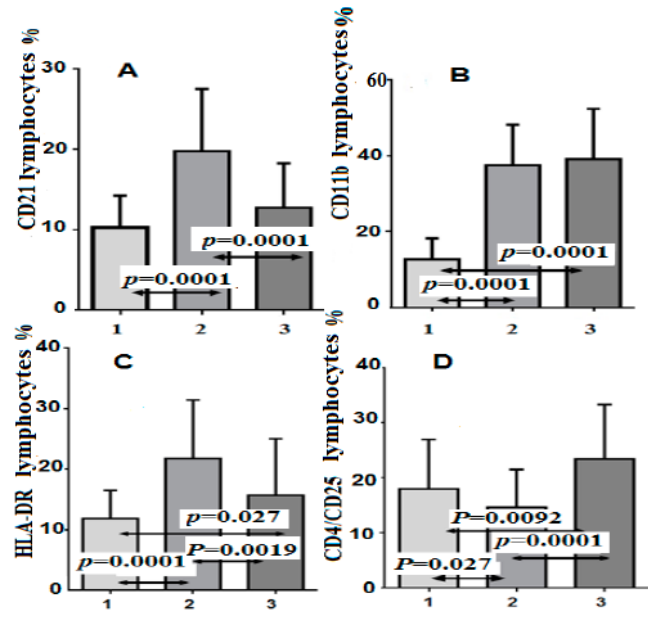

Figure 8: Changes in the relative content of $\mathrm{B}$ lymphocytes (A), lymphocytes with the expression of integrin molecules LFA-1 (B), HLA-DR+ antigens (C), and T-regulators (D).

1 - before surgery, 2 - 1 day after the surgery, $3-7$ days after the surgery.

This phenomenon can be explained by the possible release into the peripheral blood of the socalled B-regulatory lymphocytes, which by producing IL10, contribute to significant suppression of the inflammatory process $[23,30]$, which in this case can be observed during the surgical intervention.

An increase in the relative content of CD11b lymphocytes with the expression of cell adhesion and migration receptors (LFA-1 integrins), phagocytosis of bacteria and particles opsonized by the complement component C3bi [31], (Fig. 8B) on day one $и 7$ $(p=0.0001)$ after the surgery was established. At the same time, an increase in \% of lymphocytes with HLA$\mathrm{DR}+$ antigen expressed (Fig. 8-2C, $p=0.0001$ and 8-3C, 
$p=0.027)$ was noted. In this regard, it is important that the function of HLA-DR antigens means participation in the presentation of antigens to T-cells during the immune response development to various pathogens and non-infectious antigens. It should be noted (Fig. 8D) that the count of T-regulatory lymphocytes significantly increased $(C D 4+C D 25+, p=0.009)$ by the time when patients were discharged; it can presumably reflect a decrease in the hyperactivation of the immune system, which begins on day 7 , after the presumably rapid development of the postoperative autoimmune process (CD4+CD25+ deficiency, $p=0.027)$.

Therefore, during CABS under CPB, both innate and adaptive immunity are activated, which should be taken into account in the selection of immunomodulatory drugs. d) Analysis of immunity activation indices in patients with $C A B S$ under $C P B$

The section includes a study of activation indices reflecting the state of immunity in patients after CABS under CPB. The data provided in Fig. 9A indicates that the average value for the group of patients with the LII index (leukocyte intoxication index) most effectively reflects the state of endogenous intoxication of the body, which was high, which is determined by a special calculation formula using the patients' peripheral blood cells [32]. Its value increased in all time points in the postoperative period $(p=0.0001$ and $p=0.02$ ).
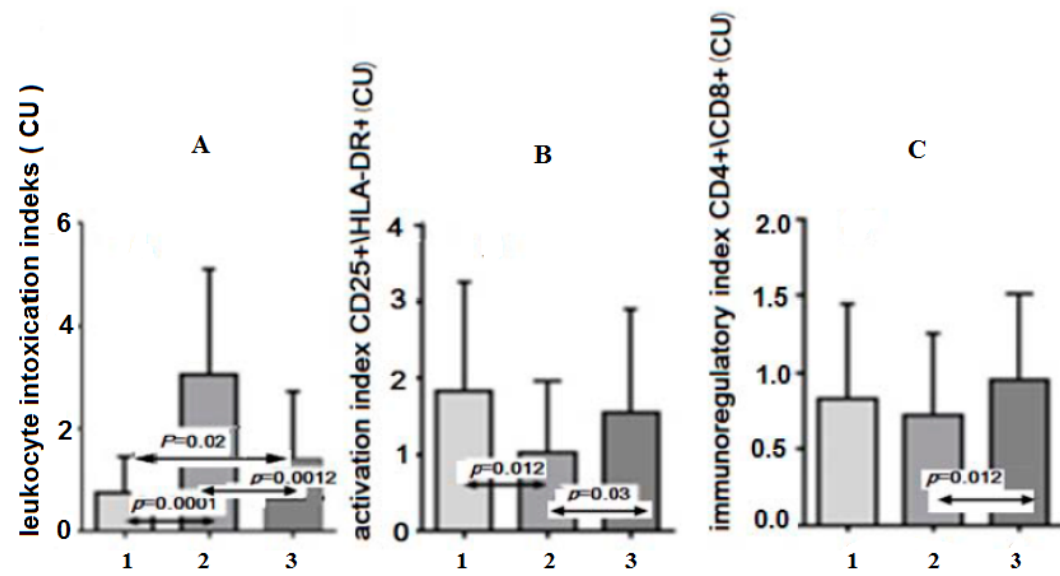

Figure 9: Activation indices change in patients after CABS under CPB.

A - LII (CU); B - CD25+/HLA-DR+ (CU); C- immunoregulatory CD4+/CD8+ (CU).

1 - before surgery, 2 - 1 day after the surgery, 3-7 days after the surgery.

A dynamic analysis of the activation indices CD25+/HLA-DR+ showed a significant decrease in the parameter $(p=0.012)$ on the first day after the surgery (Fig. 9B), followed by recovery on the 7th day after the surgery. The stated index is very significant because it reflects the development of the depletion of the functional reserve of the immune response [40]. We can consider that its normalization on the 7th day after the surgery should be regarded as a positive phenomenon. At the same time, changes in the immunoregulatory index CD4+/CD8+, which reflect a severe inflammatory process with its values well below 1.0, did not reveal any significant changes on the 1st and 7th day after the surgery compared to the preoperative period (Fig. 9C), which most likely indicates severe and more local inflammation after CABS under CPB. Nevertheless, all the detected changes once again confirm the significant role of adaptive immunity in CABS operations under CPB. e) Analysis of humoral immunity factors (immunoglobulins) in patients after CABS under $C P B$

Since immunoglobulins $\lg \mathrm{G}$, IgM and $\lg A$ determine the protection of the human body from various pathogens (bacteria, viruses, and other foreign substances), their determination in the serum of patients after CABS under CPB is a medical necessity. Fig. 10 shows the quantitative content of $\lg G, \lg M$, and $\lg A(g \mid L)$ in the serum of patients before the surgery and on days 1 and 7 after it. 

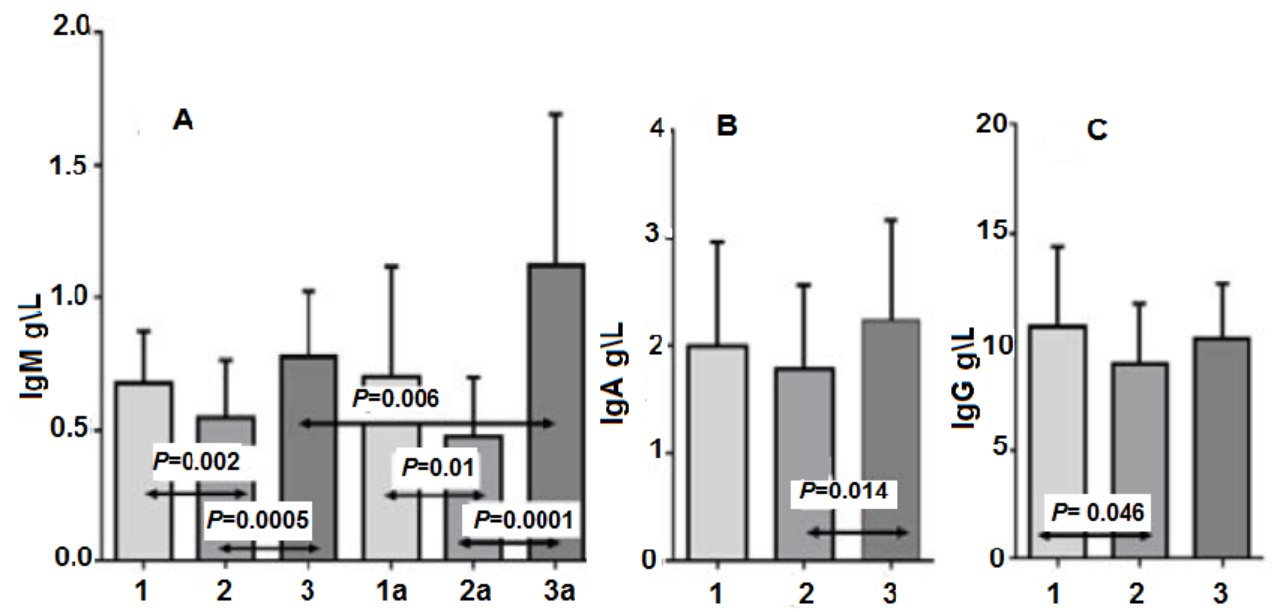

Figure 10: The average absolute content $(\mathrm{g} \mid \mathrm{L})$ of $\lg \mathrm{G}(\mathrm{A})$, $\lg A(B)$, and $\lg M(\mathrm{C})$ immunoglobulins in blood serum in patients after CABS under CPB.

1 - before surgery, 2 - 1 day after the surgery, 3-7 days after the surgery, 1a - 2a - 3a - patients receiving Immunovenin.

As it turned out, a significant decrease of an average level of immunoglobulins in patients on the 1st and normalization on day seven after the surgery concerned only $\operatorname{lgG}$ and $\operatorname{lgM}$ classes, whereas the change in IgA level was not reliable (Fig. 10B). The most significant drop in concentration $(p=0.002)$ was observed in IgM immunoglobulin, the most important key immunoglobulin, since it is firstly produced by the body in response to the introduction of a pathogen and has a significantly higher valency and avidity compared to IgG. Positive is the fact that IgM and IgG levels are completely restored (only by average values of protein levels for a group of patients!) by the time when patients are to be discharged.

f) The use of the officinal $\lg G$ preparation (Immunovenin) in patients with CABS under CPB

It is very important to note that the studies performed (see above) resulted in serious negative changes revealed in the state of the immune system in patients having undergone CABS under CPB; the changes predominantly persisted at the time of their discharge. These include severe leukocytosis, granulocytosis, and monocytosis, a sharp increase in stab neutrophils, oxidative stress of phagocytes, and endogenous intoxication. Negative changes also affected the adaptive immunity and manifested as a natural killer deficiency, lymphocytes, cytotoxic/ suppressor T-cells, and other disorders. Therefore, for immunocorrection, studies were performed with a small number of patients involved $(n=18)$ and concerned the officinal powerful lgG immunotropic drug, i. e. Immunovenin, that, according to our previously obtained data on gabriglobin (Immunovenin analog), levels many of the above immune parameters in patients $(42,43)$.

It turned out that the administration of Immunovenin was characterized by a significant effect exerted mainly on indicators of innate immunity state in patients. So, once Immunovenin was administered, the content of leukocytes, granulocytes, and monocytes by day 7 was significantly (and reliably) lower than that in patients not receiving the drug (Fig. 1A, B, D).

It is of importance that the application of Immunovenin resulted in the number of stab neutrophils reaching the preoperative level on day 7 (Fig. 1C, values in $1 \mathrm{a}$ and $3 \mathrm{a}$ charts, $p=0.07$ ), while in the group without receiving Immunovenin their content remained significantly increased (with the differences remaining highly significant, $p=0.0001$ ). No similar result was found when determining the content of granulocytes and monocytes.

The number of stab neutrophils reduced back to normal is a positive prognostic sign since an increase, and prolonged circulation of this cell type in the bloodstream is considered an unfavorable sign. It is associated with impaired cardiac activity [27]. Of similar importance are decreased markers of the inflammatory process in patient's body (total leukocytes, granulocytes, and monocytes, respectively $P=0.008$; $=0.006$; $=0.02$; Fig. $1 \mathrm{~A}, \mathrm{~B}, \mathrm{D})$, which indicates a significant decrease in the intensity of the inflammatory process in patients than underwent CABS under CPB and were treated with an immune preparation. This observation can be considered fundamental for the corrective immunotropic therapy given to cardiosurgical patients, including the therapy applied for their rehabilitation.

In patients receiving Immunovenin, on the 7 th day after the surgery, the number of cells with CD40 membrane expression significantly decreased $(p=0.013)$ compared to the untreated patients (Fig. 2C, group 4). Such a reaction of cells also can reflect the positive effect of immunoglobulin on the course of the inflammatory process after the surgery.

We calculated the relative number of patients with increased LII (>1.5 UE) in the groups which received the immunoglobulin $G$ preparation 
(Immunovenin) and did not receive the same (Fig. 11). It turned out that after the administration of the drug to patients, their number decreased much more than those who did not receive the drug, and the difference turned out to be highly reliable $\left(\chi^{2}=30.57, p<0.002\right)$.
Therefore, the administration of Immunovenin after CABS under CPB is accompanied by a significant decrease in the endogenous intoxication of the body, which is of great importance for maintaining the health of the operated patients.

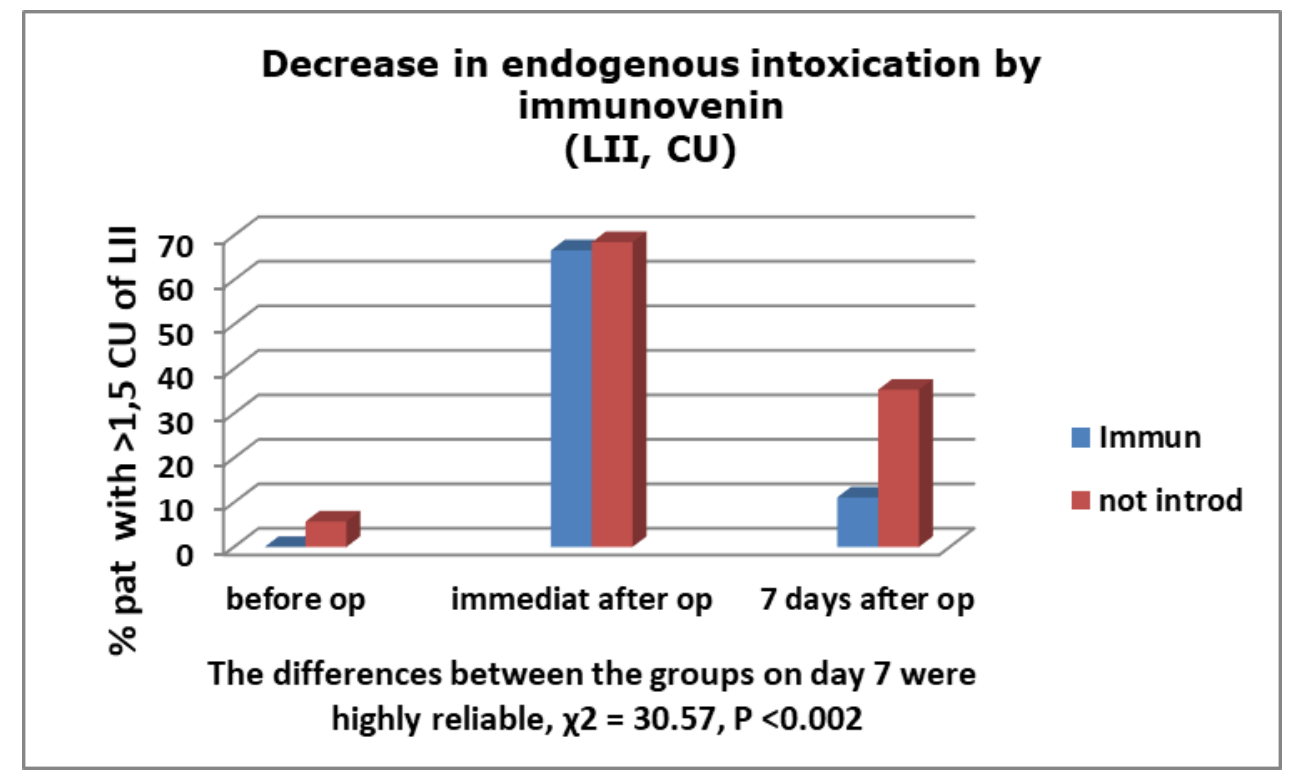

Figure 11: Decrease in endogenous intoxication (LII-conventional units-CU) in patients after CABS under CPB who received immunosuppression therapy with Immunoveninin (IgG preparation).

Designations: op - operation, Immune - Immunovenin, not introd - not administered, immediate - immediately after the surgery, pat - patients

Finally, a significant increase was revealed in the IgM norm in the group of patients receiving Immunovenin (Fig. 10.3a, $p=0.006$ ), which may be a compensatory immunotropic-related reaction after a pronounced protein deficiency developed immediately after the surgery.

However, immuno-replacement therapy with Immunovenin in the case of IgG deficiency in patients after CABS under CPB did not have a significant effect on eliminating its deficiency, since it returned to normal by the 7 th day in the group under conventional treatment when calculating the average protein content. Moreover, when calculating the number of patients with $\lg \mathrm{G}$ deficiency on the $7^{\text {th }}$ day of the postoperative period according to the $x 2$ criterion, there was no significant difference in the groups treated with and without Immunovenin $(\alpha 2=1.19, p>0.05)$. However, the positive effect of Immunovenin in patients after CABS under CPB in the number of patients with IgG deficiency nevertheless was observed, since, on the 1st day of the postoperative period, the deficiency was detected in $60 \%$ of patients. After the application of Immunovenin, their number decreased to $6.67 \%$. I.e., it decreased nine times, while in the group without Immunovenin, the number of patients with IgG deficiency in the same period remained the same: $22.2 \%$. Therefore, we can conclude that with such changes in the blood immunoglobulins of patients as a result of surgery,
Immunovenin should be administered during CABS under CPB. Moreover, it should be administered probably even before the surgery, which will help stabilize their concentration in the bloodstream during the surgery. However, of course, this possibility requires experimental confirmation and more extensive clinical material.

To judge how Immunovenin affects the adaptive immunity state in patients after CABS under CPB, the obtained data were insufficient, and therefore further studies are required in the long run.

\section{General Discussion}

A significant role in the heart pathology is firmly established to be attributed to the innate immunity cells, i.e., neutrophils and monocytes $[1,3,34,35]$. During heart surgery, the activation of this cell link is registered, which is well shown with laboratory tests. Thus, within this study, we found that neutrophils and monocytes are subject to the greatest quantitative and qualitative changes when performing surgical interventions on the heart. If, on day seven, after the surgical intervention, the features of adaptive immunity cells return to normal, the functions of neutrophils and monocytes at this time are still far from the preoperative values. This observation is extremely important as similar changes in the immune status can later turn out to be pathologically altered in 
some of the discharged patients and be accompanied by several serious complications. Regarding this possibility, the discharged patients are to be monitored for several months, initially, by filling out a special questionnaire to identify various complications in patients having undergone the surgery. In the future, it will become clear what needs to be done to prevent them.

During the study, for the first time we were able at a heart surgery to establish quantitative and functional changes in key cells of the innate immunity with expression in the neutrophils of CD64, CD16, and CD40 membrane markers, and in monocytes, i.e., CD4, CD16, CD56, and HLA-DR. The mentioned cell markers are actively involved at various stages of the body's immune defense and reflect its features before the surgery and on day one and day seven after it. So, though it was previously considered that the high-affinity $\mathrm{FC}_{\gamma}-\mathrm{I}$ receptor (CD64) or the neutrophils, carrying it, increased mainly during the infectious process or sepsis, it turned out that such processes occurred with neutrophils in patients having undergone CABS under CPB. Since the inflammatory process caused by surgery usually occurs under aseptic conditions, we can assume that CD64 neutrophil membrane marker should be classified less as a "septic" marker, more as a stress-induced antigen. This observation is undoubtedly fundamental and will contribute to the development of new directions in immunotropic therapy regarding clinical immunology in patients having undergone heart surgery. Nevertheless, against the background of an infectious process, especially a severe one, the CD64+ marker is certainly still a "septic" marker of pathology, which we clearly showed $[36,37]$.

Similar considerations should be applied to neutrophil CD16 receptors, HLA-DR, and monocyte CD16 receptors, the changes in expression of which depend significantly on a result of the surgery.

For the first time, we also found an increase in the content of CD40+ neutrophils. It was observed on the first day after the surgery and then increased significantly on day 7 . This phenomenon can be interpreted as a positive one since neutrophils are known to actively participate in the immune response $[6,7]$ and thrombolysis [38]. This phenomenon can also cause a negative effect because binding to the CD40 ligand can cause negative processes within the body [39]. Since the CD40 receptor is the 5th member of the TNFRSF5 receptor superfamily, it is possible that excess TNF levels can be increased [40]. In patients having undergone CABS, Immunovenin administered on day 1 of the postoperative period had the dramatic importance for the correction of the innate immunity. In this case, there was a significant decrease in the number of neutrophils (the stab neutrophil population reached normal values as compared to patients not receiving the drug). A significant decrease in monocytes was also observed on day seven after the intervention. The results obtained indicate that the drug has anti-inflammatory properties. In domestic and foreign literature, no such approach is found. It must be noted that since several patients had pre-operative neutrophil activity due to the oxygen radicals production and CD40 expression, it may be advisable to administer Immunovenine to patients in the post-operational period. Here, we must add that $15 \%$ of the patients having undergone a surgical intervention had an IgG blood deficiency, and $33 \%$ of them had an IgM deficiency. Interesting and, in a certain sense, positive data were obtained regarding the calculated dynamics in the number of patients suffering from IgG deficiency who received Immunovenin injection as compared to the group not having to receive the drug; the deficiency was not detected when determining the average content of immunoglobulins in the corresponding groups of patients. Following this processing of results, the decrease in the number of patients suffering from IgG deficiency was much lower in the drug infusion group than that in the group of patients not having received Immunovenin. Once administered to patients, Immunovenin significantly increased $\operatorname{lgM}$ in them on day 7 of the postoperative period. This is a positive fact, and it most likely reflects the compensatory reaction of the humoral immune link, since IgM provides early protection of the body from bacterial agents. In patients having undergone surgery and not having received the drug, no significant increase in IgM was detected.

Adaptive immunity cells are also subject to change during heart surgery. So, studies [21,24] show that the functional ability of the T-cell link changes significantly during cardiac surgery, while the response to PPD (Tuberculin PPD - Purified Protein Derivative) is thus reduced. In [41], a decrease in the relative content of CD3+ lymphocytes by $9.0 \%$ and an increase in the level of CD8+ by 1.2 times were recorded, with an absolute and relative number of B-cells increased by 1.9 and 2.3 times, respectively. Another observation [24], also associated with the CABS surgery, indicated a decrease in the number of T-lymphocytes (T-helpers). In contrast, the number of suppressors/cytotoxic T-cells and B-cells in the studied blood samples was free of any changes. When comparing the cited data and the immune findings that we obtained, the work performed at our Center revealed a significant increase in natural killer cells on day 1 of the postoperative period and a decrease in the number of total CD3, CD4 (helpers/inducers), and CD8 (cytotoxic/suppressors) lymphocytes, together with an increase in the number of CD21+ B-lymphocytes. This sharp decrease in total lymphocytes and cytotoxic/suppressor T-cells after discharge may be complicated by the developed immunodeficiency. At the same time, the increase in Thelper lymphocytes (detected on day 7) probably 
occurred due to the compensatory reaction of the immune system due to a deep deficiency of this type of cells on day 1 of the postoperative period, with a simultaneous deficit noted throughout the postoperative period of CD8+ T lymphocytes.

At the same time, on day 1 of the postoperative period, not only the number of B-lymphocytes and lymphocytes with the expression of LFA-1 (CD11b+) and HLA-DR+ lymphocyte integrin molecules increased, but also the content of HLA-DR+ lymphocytes and T-regulatory cells (CD4+CD25+) increased on day 7 of the postoperative period. Immediately after the surgery, such changes in adaptive immunity cells could reflect the release of the so-called regulatory B-lymphocytes into circulation and their release of anti-inflammatory $\mathrm{IL}-10$, possibly reducing the intensity of the developing inflammation. The accumulation of lymphocytes with enhanced migration activity is also possible. Moreover, the preservation of an increased number of HLA-DR+ and CD4+CD25+ lymphocytes on day seven could speak of an increased presentation of antigens by lymphocytes and, at the same time, a decrease in the hyperactivation of the immune system that occurs shortly after the surgery. Naturally, these speculations should be proved in further studies, since they are of fundamental importance in cardiac surgery (CABS under $\mathrm{CPB}$ ).

\section{Conclusion}

In heart surgery (CABS under CPB), most of the innate and adaptive immunity indicators change significantly. The use of Immunovenin positively affects the innate immunity state. Thus, there is a potential opportunity to apply immunoglobulin preparations to relieve the acute phase in the postoperative period by activating innate immune cells for cardiac surgery and also for other surgical interventions. Further, it is planned to conduct fundamental research on this phenomenon using modern surgical technologies.

\section{References Références Referencias}

1. Frantz, S., Falcao-Pires, I., Balligand, J. L., Bauersachs, J., Brutsaert., D. et al. 2018. The innate immune system in chronic cardiomyopathy: A European Society of Cardiology (ESC) scientific statement from the Working Group on Myocardial Function of the ESC. Eur J Heart Fail, 20: 445-459.

2. Zhang, Y., Bauersach, J., Langer, H.F. 2017. Immune mechanisms in heart failure. Eur $\mathrm{J}$ Heart Fail, 19: 1379-1389.

3. Mann, D.L. 2015. Innate Immunity and the Failing Heart. The Cytokine Hypothesis Revisited. Circ Res, 116: 1254-1268.

4. On, Y., Taylor, J.M., Bannon, P.Cl., Geczy, C., Kritharides, L. 2005. Circulating CD10-/CDI6 low neutrophils provide a quantitative index of active bone marrow neutrophil release. $\mathrm{Br} \mathrm{J}$ Haematol, 13(1): 508-19.

5. Buckley, C.D., Ross, E.A., McGettrick, H.M., Osborne, C.E., Haworth, O.K. et al. 2006. Identification of a phenotypically and functionally distinct population of long-lived neutrophils in a model of reverse endothelial migration. J Leuko Bio, 792: 303-11.9.

6. Davis, R.E., Sharma, S., Conceição, J., Carneiro, P., Novais, F. et al. 2017. Phenotypic and functional characteristics of HLA-DR+ neutrophils in Brazilians with cutaneous leishmaniasis. J Leukoc Biol, 101: 739-749.

7. Cai, S., Kandasamy, Rahmat, J.N., Tham, S.M, Bay, B.H. et al. 2016. Lactobacillus rhamnosus. GG activation of dendritic cells and neutrophils depends on the dose and time of exposure. J Immunol Res, 2016: 8.

8. Horckmans, M., Ring, L., Duchene, J., Santovito, D., Schloss S. et al. 2017. Neutrophils orchestrate post-myocardial infarction healing by polarizing macrophages towards a reparative phenotype. Eur Heart J, 38: 187-197.

9. Vono, M., Lin, A., Norrby-Teglund, A., Koup, R.A., Liang, F., Loré, K. 2017. Neutrophils acquire antigen presentation capacity to memory CD4+ T cells in vitro and ex vivo. Blood, 129: 1991-2001.

10. Araújo, H.A., Franck, G., Shvartz, E., Sukhova, G., Libby, P. 2015. TLR2 and neutrophils potentiate endothelial stress, apoptosis and detachment: implications for superficial erosion. Eur Heart J, 36:1394-1404.

11. Hoyer, F.F., Nahrendorf, M. 2017. Neutrophil contributions to ischaemic heart disease. European Heart Journal, 38: 465-472.

12. Merino, A., Buendia, P.A., Martin-Malo, A.F. et al. 2010. Senescent CD14+CD16+ monocytes exhibit proinflammatory and proatherosclerotic activity. J Immunol, 186: 1809-1815.

13. Weber, C, Shantsila, E., Hristov, M., Caligiuri, G., Guzik, T. et al. 2016. Role and analysis of monocyte subsets in cardiovascular disease Joint consensus document of the European Society of Cardiology (ESC) Working Groups "Atherosclerosis \& Vascular Biology" and "Thrombosis". Thromb Haemost, 116: 626-637.

14. Shahid, F., Lip, G.Y., Shantsila, E. 2018. Role of monocytes in heart failure and atrial fi brillation. $J$ Am Heart Assoc, 7: 167-304.

15. Wrigley, B.J., Shantsila, E., Tapp, L.D., Lip, G.Y. 2013. CD14++CD16+ monocytes in patients with acute ischaemic heart failure. Eur J Clin Invest, 43: 121-130.

16. Suzuki, A., Fukuzawa, K., Yamashita, T., Yoshida, A., Sasaki S. et al. 2017. Circulating intermediate CD14++CD16+ monocytes are increased in patients with atrial fibrillation and reflect the 
functional remodelling of the left atrium. Europace, 19: 40-47.

17. Tapp, L.D., Wrigley, B.J., Pamukcu, B., Lip, G.Y. 2012. The CD14++CD16+ monocyte subset and monocyte-platelet interactions in patients with STelevation myocardial infarction. J Thromb Haemost, 10: $1231-41$.

18. Rothe, G., Gabriel, H., Kovacs, E., Klucken, J., Stöhr, J., Kindermann, W., Schmitz, G. 1996. Peripheral blood mononuclear phagocyte subpopulations as cellular markers in hypercholesterolemia. Arterioscler Thromb Vasc Bio, 16: 1437- 1447.

19. Kazimierczyk, E., Eljaszewicz, A., Zembko, P., Tarasiuk, E., Malgorzata, M. et al. 2019. The relationships among monocyte subsets, miRNAs and inflammatory cytokines in patients with acute mycardial infarction. Pharmacological Reports, 71: 73-81.

20. Patel, A.A., Zhang, Y., Fullerton, J.N., Boelen, L., Rongvaux, A.S. et al. 2017. The fate and lifespan of human monocyte subsets in steady state and systemic inflammation. J Exp Med, 214: 1913-1923.

21. Sano, T., Morita, S., Tominaga, R., Masuda, M., Tomita, Y., Yasutsune, T., Yasu, H. 2002. Adaptive immunity is severely impaired by open-heart surgery. Jpn J Thorac Cardiovasc Surg, 50: 201-205.

22. Zhu, Z.F., Meng, K., Zhong, Y.C., Qi, L., Mao, X.B. et al. 2014. Impaired circulating CD4+LAP+ regulatory $\mathrm{T}$ cells in patients with acute coronary syndrome and its mechanistic study. PLoS One, 9.

23. Jiao, J., Lu, Y-Z., Xia, Ni., Wang, Y-Q., Tang, T-T. et.al. 2018. Defective Circulating Regulatory B Cells in Patients with Dilated Cardiomyopathy. Cell Physiol Biochem, 46: 23-35.

24. Markewits, A., Lante, W., Franke, A., Marohi, K., Kuhimann, W.D., Weinhold. C. 2001. Alterations of cell-mediated immunity following cardiac operation: clinical implication and open questions. Shock, 16: 10-5.

25. Caldefie-Chezet, F., Walrand, S., Moinard, C.T., Tridon, A., Chassagne, J. et al. 2002. Is the neutrophil reactive oxygen species production measured by luminol and lucigenin chemiluminescence intra or extra cellular? Comparison with DCFH-DA flow cytometry and cytochrome c reduction. Clin Chim Acta, 319: 9-17.

26. Holzer, K., Richter, A., Konietzny, P., Schübel, F., Wilhelm, K. et al. 2003. Functions of circulating and intra-abdominal polymorphonuclear leukocytes during human secondary peritonitis. Zentralblatt fur Chirurgie, 128: 291-297.

27. Kong, T., Hoon Kim, T., Seok Park, Y., Phil Chung S., Sun Lee, H. et al. 2017. Usefulness of the delta neutrophil index to predict 30-day mortality in patients with ST segment elevation myocardial infarction. Sci Rep, 16:15718. doi: 10.1038/s41598017-15878-5.

28. Zhen, A., Krutzik, S.R, Levin, B.R., Kasparian, S., Zack, J.A. et al. 2014. CD4 Ligation on human blood monocytes triggers macrophage differentiation and tnhances HIV infection. J Virol, 88. doi: 10.1128/JVI. 00616-14.

29. Zemskov, V.M., Pronko, K.N., Ionkin, D.A., Chzhao, A.V., Kozlova, M.N. et al. 2019. Immune status of pancreatic cancer patients receiving cryosurgery. Medical Science, 7:73. doi: 10.3390/medsci70600 73.

30. Zhou, C.J., Ma, F., Liao, W.J., Song, L.J., Yu, D. et al. 2019. Restoration of immune suppressor function of regulatory $B$ cells collected from patients with allergic rhinitis with Chinese medical formula Yupingfeng San. Am J Transl Res, 11: 1635-1643.

31. Lukácsip, S., Nagy-Baló, Z., Erdei, A., Bajtay, Z. 2017. The role of CR3 (CD11b/CD18) and CR4 (CD11c/CD18) in complement- mediated phagocytosis and podosome formation by human phagocytes. Immunol Lett, 189: 64-72.

32. Kalf-Caliph, Y.Y. 1941. Leukocyte index of intoxication and its practical significance. Medical business, 1: 31-35.

33. Tarasyuk, V.V., Titov, L.P., Zhmurovskaya, L.S. 2005. Methods of diagnosis of cell-molecular damage to the T-system immunity in hepatitis $\mathrm{C}$ and mixed hepatitis $\mathrm{B}+\mathrm{C}$. Achievements of medical science of Belarus, (UDC: 616.36.-002.24:578.891] - 097), http://med.by/dmn/book.php?book=05-7_23.

34. Yang, X.J., Zhang, L., Yu, C., Yang, X-F., Wang, H. 2014. Monocyte and macrophage differentiation: circulation inflammatory monocyte as biomarker for inflammatory diseases. Biomarker Res, 2: 2-9.

35. Italiani, Y.P., Boraschi, D. 2014. From monocytes to M1/M2 macrophages: Phenotypical vs functional differentiation. Frontiers in Immunol, 5: 514.doi: 10.3389/fimmu.2014.00514.

36. Zemskov, V.M., Alekseev, A.A., Kozlova, M.N, Shishkina, N.S., Gnatenko, D.A. et al. 2016. Immune diagnostics of septic complications in burns. Biology Bulletin Reviews, 6: 344-354.

37. Zemskov, V.M., Alekseev, A.A., Kozlova, M.N., Shishkina, N.S., Gnatenko, D.A et al. 2016. A composite biomarker panel as a highly informative and reliable tool for predicting septic complications. Jacobs Journal of Biomarkers, 2: 1-10.

38. Stief, T.W. 1991. Nonradical excited oxygen species induce selective thrombolysis in vivo. Thromb Res, 62:147-63.

39. Rizvi, M., Pathak, D., Freedman, J.E., Chakrabarti. 2008. CD40-CD40 ligand interactions in oxidative stress, inflammation and vascular disease. Trends Mol Med, 14: 530-8. 
40. Zemskov, A.M., Zemskov, V.M., Karaulov, A.V. (Ed.). 2008. The Textbook "Clinical Immunology" for students of higher vocational education. Moscow: Publishing House GEOTAR- Media, $426 \mathrm{p}$.

41. Golovkin, A.S. 2014. Mechanism of syndrome of system inflammatory response after operations with application of artificial blood circulation. The dissertation for the degree of Doctor of Medical Sciences. Kemerovo.

42. Zemskov, V.M., Alekseev, A.A, Kozlova, M.N., Shiskina, N.S., Bleykhman, D.A. et al. 2017. Changes in the immune system depending on the stage of burn disease and the area of thermal destruction. immunoglobin replacement therapy with gabriglobin. Internat $J$ Recent Scientific Research, 8(2), 15653-15662. DOI: http://dx.doi.org/ 10.24327/ijca.2017. 3404.0282

43. Zemskov, A.M., Zemskov, V.M, Zemskova, V.A., Zoloedov, V.I., Dorohov, E.V. 2017. Immunoglobulins - present and future. Internat $\mathrm{J}$ Current Advanced Research, 6(4), 3400-3404. DOI: http://dx.doi.org/10.24327/ ijcar.2017. 3404.0282 